\title{
Fenotipos del asma en Pediatría
}

\section{Phenotypes of asthma in pediatrics}

\author{
Sonia M. Restrepo-Gualteros, MD.(1); Milena Villamil-Osorio, MD.(2); \\ CARLos E. RODRÍGUEZ-MARTíneZ ${ }^{(3)}$
}

\section{Resumen}

El conocimiento de la existencia de varios fenotipos de sibilancias, junto con sus características distintivas, proporciona una visión del desarrollo del asma y de su historia natural. El asma es una enfermedad compleja con múltiples mecanismos fisiopatológicos. La identificación de estos fenotipos es importante para el estudio de las vías de desarrollo del asma y los mecanismos subyacentes de la enfermedad, para ayudar a decidir cuál es la terapia más adecuada y predecir la evolución clínica de los pacientes. El propósito de esta revisión es describir los diferentes estudios disponibles en la literatura para el abordaje y a descripción de los fenotipos de sibilancias en la infancia y sus factores de riesgo.

Palabras clave: asma, sibilancias, niños, fenotipos.

\begin{abstract}
Knowledge of the existence of various types of wheezing, together with their distinctive characteristics, gives a view of the development of asthma and its natural history. Asthma is a complex disease with multiple pathophysiological mechanisms. Identification of these phenotypes is important for the study of the developmental pathways of asthma and the underlying mechanisms of the disease, in order to better decide what the most adequate therapy is and to predict the clinical evolution of these patients. The purpose of this review is to describe the studies available in the literature, in order to approach and describe the phenotypes of wheezing in childhood and their risk factors.
\end{abstract}

Keywords: : asthma, wheezing, children, phenotypes.

El conocimiento de la existencia de varios fenotipos de sibilancias, junto con sus características distintivas, proporciona una visión del desarrollo del asma y de su historia natural y muestra que el asma es una enfermedad compleja con múltiples mecanismos fisiopatológicos (1). La identificación de estos fenotipos es importante para el estudio de las vías de desarrollo del asma y los mecanismos subyacentes de la enfermedad, con mira a ayudar a decidir cuál es la terapia más adecuada y predecir la evolución clínica de los pacientes (2).

En el estudio respiratorio de niños de Tucson (Tucson Children's Respiratory Study, TCRS), con base en el inicio y la persistencia de las sibilancias en los participantes, los pacientes se clasificaron en 4 fenotipos de sibilancias: sibilantes tempranos transitorios, persistentes, de inicio tardío, y no sibilantes (3).

\footnotetext{
${ }^{(1)}$ Neumóloga Pediatra, Universidad Nacional de Colombia, Universidad El Bosque, Hospital de la Misericordia. Bogotá, Colombia.

(2)Neumóloga Pediatra Hospital de la Misericordia, Universidad El Bosque. Bogotá, Colombia

${ }^{(3)}$ Neumólogo Pediatra, Universidad $\mathrm{Na}-$ cional de Colombia, Universidad El Bosque, Universidad Militar Nueva Granada. Bogotá, Colombia.

Correspondencia: Sonia M. RestrepoGualteros, correo electrónico: smrestrepog @unal.edu.co

Recibido: 23/05/15. Aceptado: 01/06/15.
} 
Los participantes que experimentaron uno o más episodios sibilantes antes de la edad de 3 años, con resolución antes de los 6 años de vida, se clasificaron como sibilantes tempranos transitorios. Los que no presentaron sibilancias antes de los 3 años pero sí las presentaron entre los 3 y los 6 años, como sibilantes de inicio tardío; aquellos que presentaron episodios sibilantes antes de los 3 años y persistieron con dichos episodios a los 6 años de vida, como sibilantes persistentes, y el resto de participantes se clasificaron como no sibilantes (3).

Se identificaron factores de riesgo para cada uno de estos fenotipos: mientras que el tabaquismo materno se asoció con el fenotipo sibilante temprano transitorio; el asma materna, el sexo masculino y la presencia de rinitis alérgica durante el primer año de vida se asociaron con el fenotipo sibilantes de inicio tardío. El asma materna, el tabaquismo materno, la presencia de rinitis alérgica, la dermatitis atópica durante el primer año de vida y el sexo masculino fueron predictores independientes del fenotipo sibilante persistente. El hecho de que cada uno de los fenotipos tenga distintos factores de riesgo sugiere que cada fenotipo refleja entidades fisiopatológicas diferentes. Luego de que fueran practicadas las pruebas cutáneas de alergia a los 6 años de vida, el TCRS realizó una clasificación adicional de los fenotipos de sibilancias en 3 categorías: sibilantes tempranos transitorios, no atópicos, $\mathrm{y}$ asma/sibilancias asociadas a inmunoglobulina E (Ig E), con base en los resultados del test de reto a metacolina, la variación diurna del flujo espiratorio pico, y las respuestas a un cuestionario que indagaba la presencia o ausencia de sibilancias/atopia (4).

En el estudio de la isla de Wright, así como en el estudio multicéntrico de alergia (The Multicenter Allergy Study, MAS) y en el de Manchester de asma y alergias (The Manchester Asthma and Allergy Stu$d y$, MAAS) se adoptaron clasificaciones similares a la del TCRS, pero los criterios en cuanto a la edad fueron diferentes: mientras que en el de la isla de Wright se realizaron evaluaciones a los 4 y 10 años (5), en el MAS se realizaron a los 3 y a los 7 años y en el MAAS a los 3 y 5 años de edad. Los predictores de sibilancias persistentes fueron la historia familiar de asma, las infecciones respiratorias recurrentes a edades tempranas y la sensibilización alérgica.
La presencia de sibilancias en la niñez tardía fue el criterio para clasificar a los participantes. En el estudio de la isla de Wright, los participantes que aún presentaban episodios sibilantes a los 10 años de vida se sub clasificaron como sibilantes atópicos y no atópicos, cada uno de ellos con diferentes factores de riesgo; mientras que la presencia de asma en los hermanos, la dermatitis atópica en el primer año de vida, la rinitis a la edad de 4 años y el sexo masculino fueron más prevalentes en los sibilantes atópicos, el asma materna y las infecciones respiratorias recurrentes a los 2 años de vida fueron más prevalentes en los sibilantes no atópicos (6).

En el MAS, la presencia de sibilancias a la edad de 13 años se utilizó para subclasificar a los pacientes, y se relacionó con la atopia en los padres, la atopia en el paciente, los niveles elevados de IgE en edades tempranas y la exposición excesiva a alergenos intradomiciliarios (7). En el MAAS se incluyó la medición de resistencia de las vías respiratorias (sRaw) a edades tempranas, y se tuvo en cuenta como un factor de riesgo para la persistencia de sibilancias a los 5 años de vida.

En los estudios ALSPAC y PIAMA, los autores emplearon el sistema de clasificación utilizado en el TCRS, pero con análisis latentes longitudinales de clases, agregaron otros subgrupos. Mientras que en el estudio ALSPAC se describieron los fenotipos denominados sibilantes tempranos prolongados (asociados con asma materna, función pulmonar disminuida, leve hiperreactividad bronquial, pero no con atopia) y sibilantes de inicio intermedio (asociados con atopia, hiperreactividad bronquial severa, función pulmonar muy disminuida), en el estudio PIAMA solo se adicionó el fenotipo sibilantes de inicio intermedio. En ambos estudios, cada fenotipo tenía características similares, no solo en prevalencia, sino también en relación con sensibilización atópica, función pulmonar e hiperreactividad bronquial (8).

Los trabajos mencionados han aumentado el conocimiento y han permitido entender mejor los mecanismos y la historia natural de las sibilancias durante los primeros años de vida, además de los factores de riesgo para la persistencia de síntomas de asma; así mismo, han proporcionado gran ayuda para predecir 
parámetros de función pulmonar. Sin embargo, clasificar a un determinado paciente con sibilancias recurrentes en uno de estos fenotipos en la práctica clínica diaria es muy difícil, si no imposible, ya que se requiere hacerlo en forma retrospectiva (9).

Por tal motivo, un sistema de clasificación basado en síntomas propuesto en un consenso acerca de sibilancias en preescolares realizado por la Sociedad Respiratoria Europea (European Respiratory Society, ERS), que se ha considerado más útil para aplicarse en la práctica clínica diaria; divide a los preescolares sibilantes en dos grupos: uno denominado sibilancias inducidas por virus (Episodic Viral Wheeze, EVW) y otro denominado sibilancias de múltiples desencadenantes (Multiple-Trigger Wheeze, MTW) (10).

De acuerdo con esta definición, el fenotipo EVW se refiere a niños que presentan exacerbaciones exclusivamente desencadenadas por infecciones virales respiratorias sin evidencia de síntomas entre los episodios. Por el contrario, el fenotipo denominado MTW se refiere a niños que sibilan en respuesta no solo a infecciones virales respiratorias, sino también a otros desencadenantes tales como alergenos, actividad física, cambios de clima o exposición al humo de cigarrillo (11).

Dicha clasificación, inicialmente se consideró pragmática y útil en la práctica clínica diaria para pacientes preescolares con sibilancias recurrentes y con potenciales implicaciones terapéuticas. Mientras que dosis bajas a moderadas de esteroides inhalados en forma continua han mostrado eficacia en pacientes con MTW, no ha sido así en pacientes con EVW; en estos últimos se ha descrito que el montelukast y las dosis altas intermitentes de esteroides inhalados podrían tener un papel en su tratamiento. Sin embargo, en la actualidad se considera que hay poco soporte para el sistema de clasificación EVW/MTW, y es probable que cambie cuando aparezca evidencia adicional al respecto.

Dada no solo la importancia de establecer los fenotipos de asma, sino sus consecuencias a largo plazo se ha encontrado compromiso de la función pulmonar de niños con sibilancias en la edad adulta. Los resultados en el Estudio de Cohorte de Atopia de Melbourne, en donde se siguieron niños que nacieron con alto riesgo de alergia, se registraron sus síntomas respiratorios y se les tomó espirometría a los 12 y 18 años, demostraron que de los fenotipos tempranos (nunca/ infrecuentes, transitorios tempranos, tempranos persistentes, intermedios y los de presentación tardía), los sibilantes transitorios no tenían ninguna secuela respiratoria a los 18 años, los de fenotipo persistente tenían una reducción del $\mathrm{VEF}_{1}$ sostenida durante el todo tiempo hasta los 18 años y los de aparición intermedia tenían una limitación irreversible al flujo aéreo a los 18 años (12).

En el último año se ha propuesto un nuevo fenotipo de asma en mujeres con obesidad y menarquia temprana, a causa de la influencia hormonal e inflamatoria de las dos condiciones (13).

\section{Factores de riesgo}

Un gran número de estudios han demostrado que la aparición de los diferentes fenotipos de asma en la infancia es multifactorial y resulta de la interacción de factores genéticos y ambientales (4-15).

Se ha logrado establecer que cambios tempranos en la microbioma intestinal inducidos por el uso temprano de antibióticos puede favorecer el desarrollo de asma y otras condiciones alérgicas. De la misma manera, se ha encontrado que niños con bajo nivel socioeconómico tiene mayor riesgo de tener sibilancias persistentes (16).

La contaminación ambiental y la polución aumentan el número de ingresos hospitalarios por síntomas respiratorios y crisis. La exposición al humo de tabaco tanto en la vida fetal, como en la extrauterina, incrementa el riesgo de sibilancias tempranas (17).

Hasta ahora se han descrito múltiples polimorfismos en genes que regulan mecanismos de respuesta inmune y el desarrollo de sibilancias recurrentes en la infancia. El gen del factor nuclear I/A fue descrito como candidato para asma y rinitis alérgica (18).

Estudios de esputo en pacientes adultos ha demostrado avances en el hallazgo de biomarcadores que puedan identificar los fenotipos inflamatorios en asma (eosinofílico versus neutrofílico) y de esta manera predecir la respuesta a la terapia con corticoides 
inhalados; así que se requieren estudios de este tipo en la población pediátrica (19).

Variantes del gen protocaderina 1, importante en los procesos de adhesión celular, aumentan el riesgo de desarrollo de asma en la infancia temprana. Polimorfismos de nucleótido único (SNP, por su sigla en inglés de Single Nucleotide Polymorphism) se han relacionado con la presencia de sibilancias; el rs10063472 SNP se asoció con sibilancias transitorias tempranas y el rs6888135 SNP se identificó en pacientes que presentan síntomas de asma desde el nacimiento hasta los 7 años. En estudios realizados recientemente se ha asociado el rs10044254 SNP con una mejor respuesta a corticoides inhalados en pacientes pediátricos. También se ha encontrado que variaciones en la respuesta de endotoxinas están relacionados con asma y atopia en la infancia $(18,20)$.

\section{Conclusiones}

Los estudios en niños que presentan sibilancias han permitido describir los diferentes fenotipos del asma durante la infancia, sus características clínicas, y temporalidad, además de establecer correlación de su evolución hasta la edad adulta.

Por tanto, es vital establecer factores de riesgo cruciales en el abordaje de estos pacientes, y plantear la posibilidad de emprender hoy en día estudios genéticos que permitan conocer con antelación la respuesta a intervenciones.

\section{Conflictos de interés}

Los autores declaran no tener conflictos de interés.

\section{Bibliografía}

1. Von Mutius E. Trajectories of childhood wheeze. J Allergy Clin Immunol. 2011;127:1513-4.

2. Hultz A, Brand PL. Episodic viral wheeze and multiple trigger wheeze in preschool children: a useful distinction for clinicians? Paediatr Respir Rev. 2011;12:160-4.

3. Martínez FD, Wright AL, Taussig LM, Holberg CJ, Halonen M, Morgan WJ. Asthma and wheezing in the first six years of life. The Group Health Medical Associates. N Engl J Med. 1995;332(3):133-8.

4. Stein RT, Holberg CJ, Morgan WJ, Wright AL, Lombardi E, Taussig L, Martinez FD. Peak flow variability, methacholine responsiveness and atopy asmarkers for detecting different wheezing phenotypes in childhood. Thorax. 1997;52:946-52.

5. Kurukulaaratchy RJ, Fenn M, Matthews S, Arshad SH. Characterization of atopic and non-atopic wheeze in 10 year old children. Thorax. 2004;59:563-8.

6. Lau S, Illi S, Sommerfeld C, Niggemann B, Volkel K, Madloch C, Gruber C, et al. Transient early wheeze is not associated with impaired lung function in 7-yr-old children. Eur Respir J. 2003;21:834-41.

7. Matricardi PM, Illi S, Gruber C, et al. Wheezing in childhood: incidence, longitudinal patterns and factors predicting persistence. Eur Respir J. 2008;32:585-92.

8. Savenije OE, Granell R, Caudri D, Koppelman GH, Smit HA, Wijga A, et al. Comparison of childhood wheezing phenotypes in 2 birth cohorts: ALSPAC and PIAMA. J Allergy Clin Immunol. 2011;127:1505-12.e14.

9. Bhatt JM, Smyth AR. The management of preschool wheeze. Paediatr Respir Rev. 2011;12:70-7.

10. Brand PL, Baraldi E, Bisgaard H, et al. Definition, assessment and treatment of wheezing disorders in preschool children: an evidence-based approach. Eur Respir J. 2008; 32:1096-110.

11. Brand PL, Caudri D, Eber E, Gaillard EA, Garcia-Marcos L, Hedlin G, et al. Classification and pharmacological treatment of preschool wheezing: changes since 2008. Eur Respir J. 2014;43:1172-7.

12. Lodge CJ, Lowe AJ, Allen KJ, Zloumis S, Gurrin LC, Matheson $\mathrm{MC}$, et al. Childhood wheeze phenotypes show less than expected growth in FEV1 across adolescence. Am J Respir Crit Care Med. 2014;89:1351-8.

13. Castro-Rodríguez JA. A new childhood asthma phenotype: obese with early menarche. Paediatric Respiratory Reviews. 2016;18:85-9.

14. Wenzel SE. Complex phenotypes in asthma: Current definitions. Pulmonary Pharmacology \& Therapeutics 2013;26:7105.

15. Wahn U, Matricardi P. Toward a definition of asthma phenotypes in childhood: Making a long way shorter? J Allergy Clin Immunol 2012;130:111-2.

16. Tenero L, Piazza M, Piacentini G. Recurrent wheezing in children. Transl Pediatr 2016;5(1):31-6.

17. Gilmour MI, Jaakkola MS, London SJ, Nel AE, Rogers CA. How exposure to environmental tobacco smoke, outdoor air pollutants, and increased pollen burdens influences the incidence of asthma. Environ Health Perspect. 2006;114: 627-33.

18. Reddy M, and Covar R. Asthma phenotypes in childhood. Curr Opin Allergy Clin Immunol. 2016;16(2):127-34.

19. Diamant Z, Hanania N. The new look on asthma: linking phenotypes, endotypes and biomarkers to asthma management. Curr Opin Pulm Med. 2015;21:1-2.

20. Aspectos particulares del tratamiento de asma en niños. Capítulo 41. En: García, Caraballo. Asma: Enfoque integral y nuevas tendencias. Segunda Edición. Ed Médica Panamericana; 2014. 